\title{
Tratamento de classe III com disjuntor Haas e máscara facial de Pétit na dentadura
}

\section{mista: relato de caso}

\author{
Class III treatment with a Haas expander and Petit Face Mask on mixed-dentition: case report \\ Tratamiento de clase III con disyuntor Haas y mascarilla facial de Pétit en denticíon mixta: reporte
}

de caso

Recebido: 13/12/2021 | Revisado: 19/12/2021 | Aceito: 29/12/2021 | Publicado: 07/01/2022

\author{
Amanda Sofia Mota Martins \\ ORCID: https://orcid.org/0000-0002-1940-1256 \\ Centro Universitário Fametro, Brasil \\ E-mail: amandasoofia@outlook.com \\ Victória Gomes de Jesus Pereira \\ ORCID: https://orcid.org/0000-0003-3532-1747 \\ Centro Universitário Fametro, Brasil \\ E-mail: vitoriagomez12.vrg@gmail.com \\ Jéssica Tuane Maia Rêgo \\ ORCID: https://orcid.org/0000-0001-9396-4201 \\ Especialista em Ortodontia, Brasil \\ E-mail: tuanemaia@gmail.com \\ Nayhane Cristine da Silva de Oliveira \\ ORCID: https://orcid.org/0000-0003-2056-5853 \\ Centro Universitário Fametro, Brasil \\ E-mail: nayhane.oliveira@fametro.edu.br \\ Gabriela de Figueiredo Meira \\ ORCID: https://orcid.org/0000-0002-8285-8769 \\ Universidades Federal de Santa Maria, Brasil \\ E-mail: gabrielameira1@hotmail.com \\ Bruna Ramos Meireles dos Santos \\ ORCID: https://orcid.org/0000-0002-3796-4925 \\ Universidade Estadual Paulista "Júlio de Mesquita Filho", Brasil \\ E-mail: bruna.maisonoral@gmail.com
}

\begin{abstract}
Resumo
Objetivo: O objetivo deste artigo, por meio da metodologia de estudo de caso, é demonstrar uma das técnicas de tratamento de classe III esquelética durante a dentição mista e apresentar as vantagens do tratamento precoce. No presente caso, a paciente do sexo feminino, 9 anos de idade, após realizar os exames clínicos e radiográficos, foi comprovado a má oclusão de classe III esquelética, imediatamente foi iniciado o tratamento com o expansor Haas associado a Máscara Facial de Petit. As características da Classe III esquelética podem se tornar menos intensas com o tratamento e assim resultando em uma melhora significativa na função, aperfeiçoamento da estética e psicológico da paciente.
\end{abstract}

Palavras-chave: Má oclusão; Classe III; Técnica de expansão rápida; Maxila.

\begin{abstract}
Objective: The article's purpose, through case study methodology, demonstrate one of the skeletal class III treatment techniques during mixed dentition and present the advantages of an early treatment. In the present case, a 9-year-old female patient, after clinical and radiographic examinations, confirmed skeletal class III malocclusion and immediately started treatment with the Haas expander associated with Petit Face Mask. The characteristics of the Class III pattern may become less intense with treatment and then resulting in a significant improvement in the patient's function, aesthetic and psychological improvement.

Keyword: Malocclusion; Class III; Rapid expansion technique; Maxilla.

\section{Resumen}

Objetivo: El propósito de este artículo, a través de la metodología de estudio de caso, es demostrar una de las técnicas de tratamiento de clase III esquelética durante la dentición mixta y presentar las ventajas del tratamiento temprano. En el presente caso, una paciente de 9 años, tras exámenes clínicos y radiográficos, confirmó una maloclusión de clase III esquelética e inmediatamente inició tratamiento con el expansor de Haas asociado a la mascarilla facial de Petit. Las características del patrón de Clase III pueden volverse menos intensas con el tratamiento y, por lo tanto, dar como resultado una mejora significativa en la función del paciente, una mejora estética y psicológica.
\end{abstract}

Palabras clave: Maloclusión; Clase III; Técnica de expansión rápida; Maxilar. 


\section{Introdução}

As más oclusões estão em terceiro lugar em relação aos maiores problemas de saúde bucal no mundo, ficando abaixo somente da cárie e doenças periodontais (Cruz et al., 2019).

A oclusão é o ramo da odontologia que estuda as associações dos contatos dentários relacionados ao arco superior e inferior, envolvendo todo o sistema estomatognático, como por exemplo, os ossos e os músculos (Melo et al, 2020). A má oclusão é um dos maiores problemas de saúde bucal atualmente e sua etiologia é multifatorial, pode envolver razões ambientais, étnicas, fator genético, perdas precoce, hábitos deletérios e entre outros (Alhammadi et al., 2018).

Segundo Angle, em 1899, as más oclusões foram classificadas em relação ao primeiro molar permanente superior no sentido ântero-posterior em: Classe I (neutroclusão) perfil ortognático com maxila e mandíbula bem posicionadas e com anomalia dentária; Classe II (distoclusão) esquelética ou dentária, perfil convexo e possui divisão 1: maxila estreita com apinhamento em caninos e divisão 2: incisivos superiores lingualizados e incisivos laterais vestibularizados; e a Classe III (mesioclusão) esquelética ou dentária, perfil côncavo.

Segundo estudos, (Alhammadi et al., 2018) a prevalência global, de maloclusões apresentou-se num total de 55\% dos indivíduos, podendo ser apresentada como maior prevalência a classe I (44\%), seguida pela classe II (9\%) e classe III (5\%).

A etiologia dos tipos de má oclusão possui intervenção hereditária, pela amamentação, deglutição e por hábitos deletérios como por exemplo, a sucção digital, entre outros (Imbaud et al., 2016).

A má oclusão de classe III é a menos prevalente, é descrita como uma anomalia complexa no que se refere ao tratamento e o prognóstico, pode ser classificada como esquelética e/ou dentária. Diversos pacientes procuram tratamento devido a estética comprometida, porém, também pode envolver a função, como por exemplo, a mastigação e estruturas como, gengiva, articulações, osso. Boa parte da literatura apresenta que as características da Classe III esquelética são a protrusão mandibular e a retrusão maxilar ou os dois em conjunto, deixando o perfil côncavo. Essa anomalia que pode aumentar com a idade, por isso se faz de grande importância a sua intervenção precoce (Freitas et al., 2019).

Em relação a etiologia da má oclusão de classe III, ela pode ser hereditária (dimensões esqueléticas craniofaciais, fendas no palato/alveolares ou outras síndromes), ambiental (decorridos de hábitos deletérios, respiradores bucais, traumas, a posição para frente e para baixo da língua, hábitos posturais incorretos da mandíbula) ou uma combinação de ambas (Bedolla et al.,2018).

Para o tratamento, o correto diagnóstico se faz necessário, uma vez que são diferentes devido aos fatores esqueléticos ou dentários. A ortodontia interceptativa, é importante para correção quando o diagnóstico é realizado precocemente, antes da puberdade, já o diagnóstico tardio (após o surto de crescimento da adolescência) pode-se contar com a cirurgia ortognática. O tratamento ortopédico precoce de pacientes com essa má oclusão pode ser realizado com a máscara facial de Petit instalada após a expansão maxilar (Suassuna et al., 2018).

\section{Metodologia}

Inicialmente o responsável autorizou por meio do Termo de Consentimento Livre e Esclarecido (TCLE) em partilhar a imagem da paciente para devido fim, o artigo trata-se de um relato de caso clínico de caráter analítico descritivo, realizado na clínica odontológica do Centro Universitário Fametro, na disciplina de estágio Clínica de Atenção Integrada Infantil, do curso de graduação do CEUNI Fametro.

\section{Relado de Caso}

Paciente gênero feminino, 9 anos de idade, melanoderma, procurou juntamente com seu responsável, a Clínica de Atenção Infantil do Centro Universitário Fametro, tendo como queixa principal "mordida errada". Durante a anamnese foi verificado que a paciente apresenta uma ótima saúde em geral e não possui hábitos deletérios. 
Durante o exame extraoral, foi observado que a paciente apresenta o padrão 3 de crescimento facial, retrusão maxilar, dolicofacial e perfil côncavo (Figura 1).

Figura 1 - (A-C). Fotografia iniciais extra bucais: A) Frontal; B) Sorriso; C) Lateral.
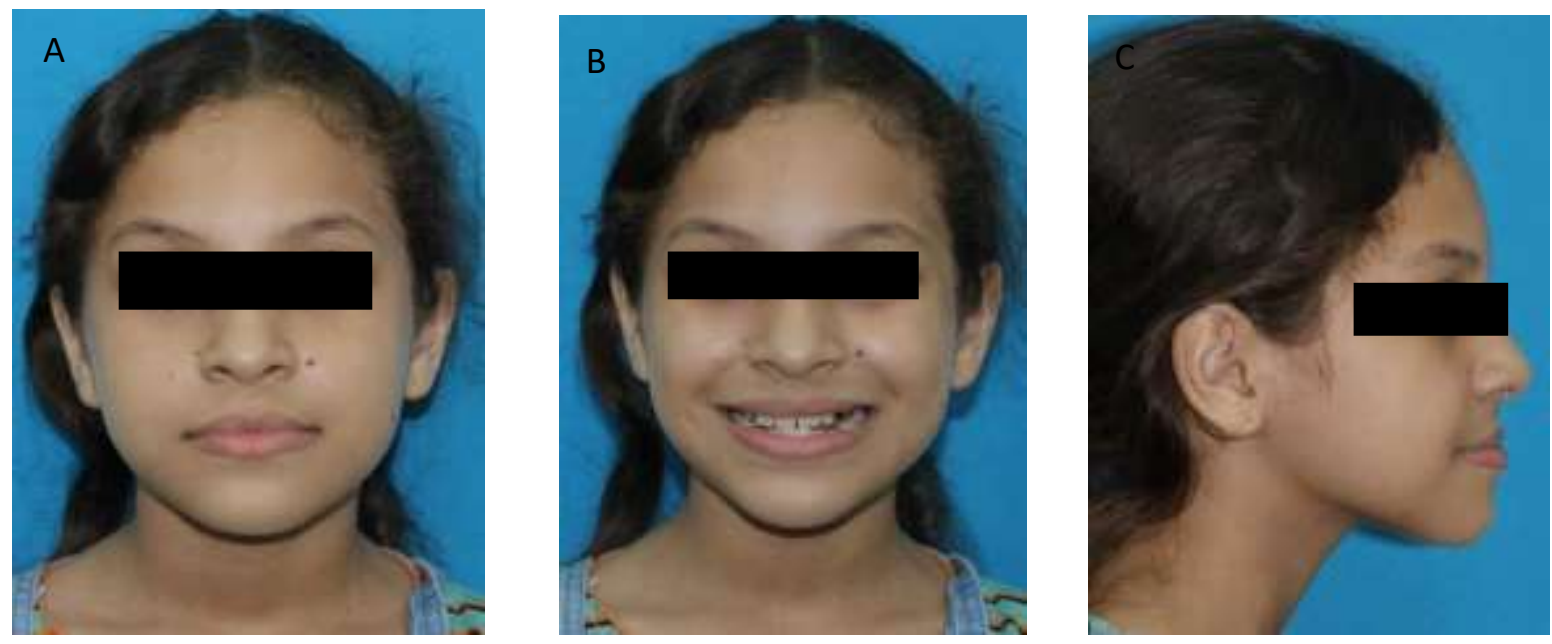

Fonte: CIMO - Clínica de Imagem (2021).

Ao exame intra-oral foi notado que a paciente estava no $2^{\circ}$ período transitório da dentadura mista, com relação de molar classe III em ambos os lados, foi observado também a falta de contato interdental de ambos os lados e mordida topo a topo. (Figura 2).

Figura 2 - (A-E) Fotografias intraorais iniciais: A) Direita; B) Frontal; C) Esquerda; D) Oclusal superior; E) oclusal inferior.
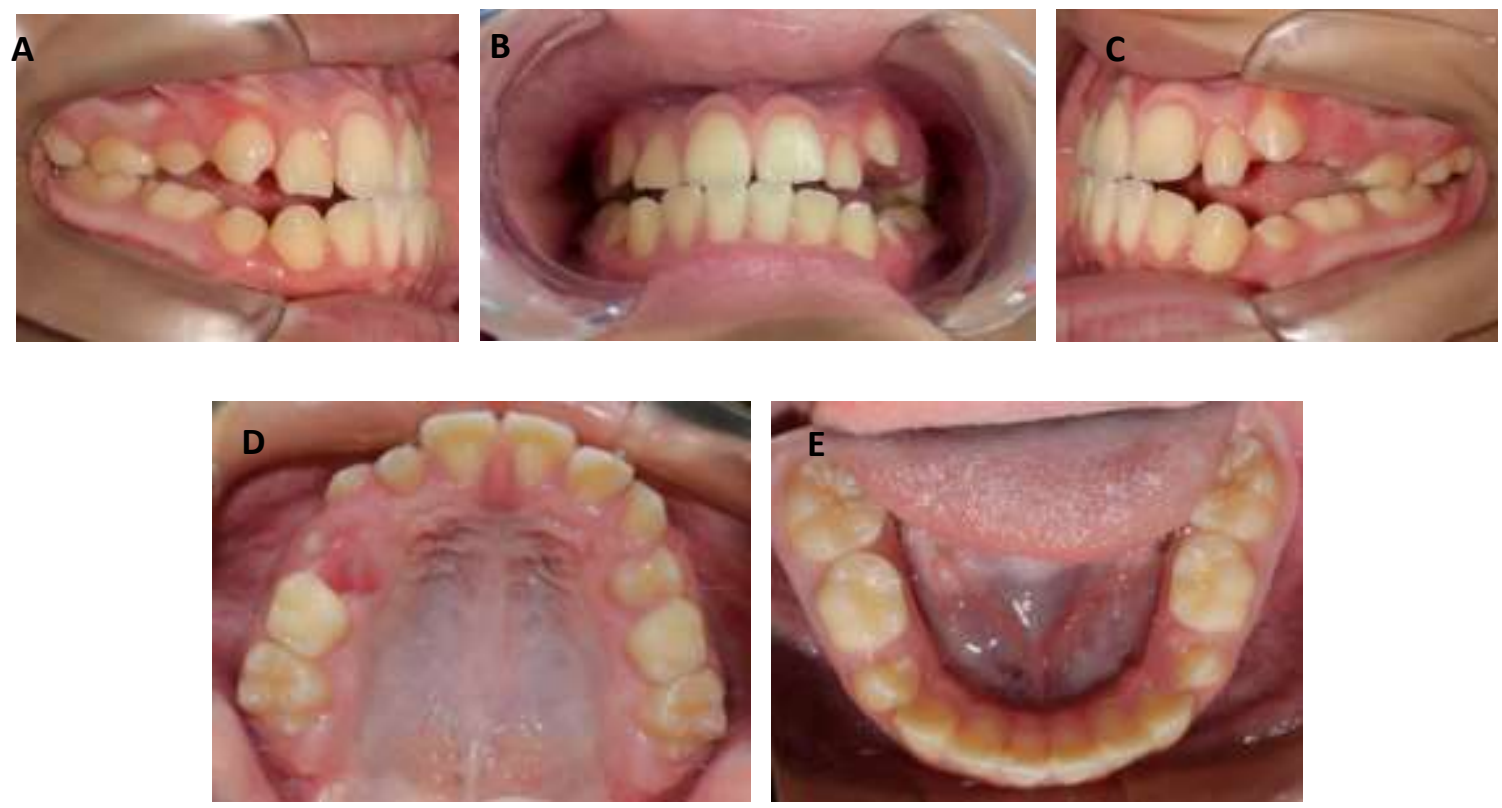

Fonte: CIMO - Clínica de Imagem (2021).

Para o início do tratamento, foi solicitado os seguintes exames; radiografia panorâmica, telerradiografia, análise cefalométrica e traçado USP. Observa-se no traçado cefalométrico de perfil (Figura 3) comprovando a protrusão mandibular pelo ângulo SNB e a retrusão maxilar pelo ângulo SNA, a combinação dos dois ângulos, o que é o valor de ANB. Nos estudos 
cefalométricos é essencial que tenha as medidas para uma melhor avaliação de possíveis tratamentos. O Quadro 1, apresenta os valores cefalométricos padrões na primeira coluna e na coluna seguinte, as medias iniciais realizadas. O diagnóstico segundo a telerradiografia foi: retrusão maxilar e Classe III esquelética.

Figura 3- Traçado cefalométrico USP inicial.

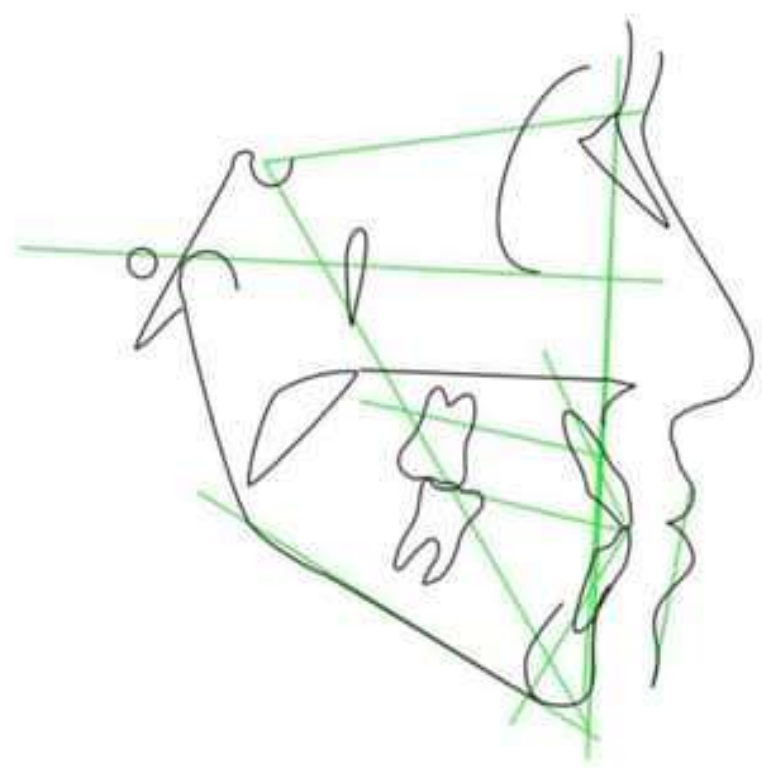

Fonte: CIMO - Clínica de Imagem (2021).

Figura 4 - Telerradiografia Lateral Inicial.

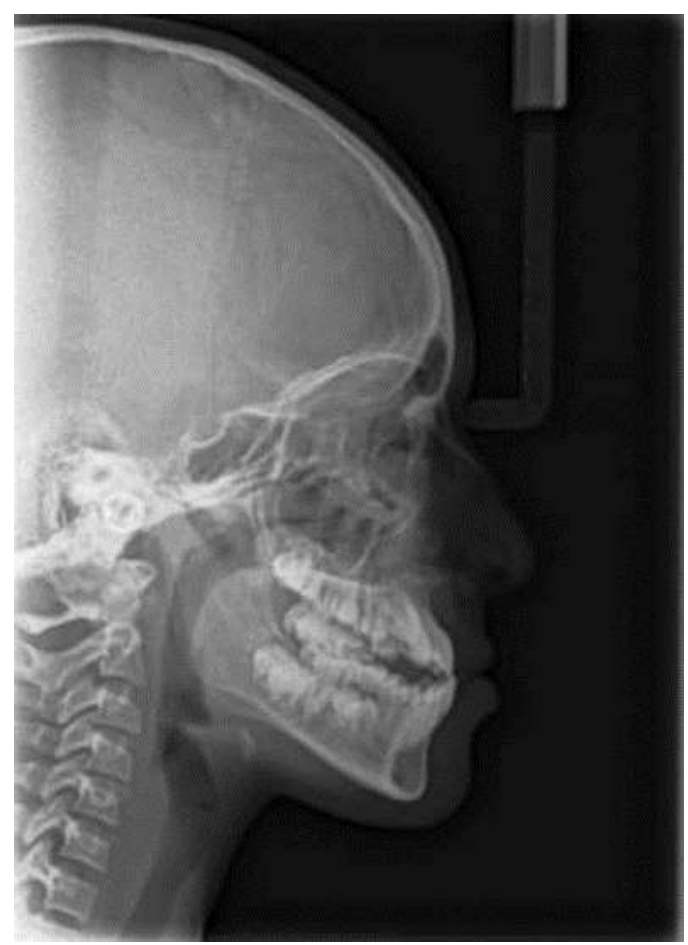

Fonte: CIMO - Clínica de Imagem (2021). 
Quadro 1 - valores Cefalométricos padrão e iniciais (análise USP)

\begin{tabular}{|l|l|l|}
\hline & Padrão & Iniciais \\
\hline SNA & $82.0 \pm 2.0$ & $80.184 \mathrm{gr}$ \\
\hline SNB & $80.0 \pm 2.0$ & $79.792 \mathrm{gr}$ \\
\hline ANB & $2.0 \pm 2.0$ & $0.392 \mathrm{gr}$ \\
\hline AND & 76.0 & $76.416 \mathrm{gr}$ \\
\hline (S-N).(Go-Me) & 32.0 & $39.178 \mathrm{gr}$ \\
\hline $1 / . \mathrm{NS}$ & 103.0 & $107.858 \mathrm{gr}$ \\
\hline $1 /$ NA & $22.0 \pm 5.0$ & $27.675 \mathrm{gr}$ \\
\hline 1/-NA & 4.0 & $4.261 \mathrm{~mm}$ \\
\hline /1.NB & $25.0 \pm 4.0$ & $28.475 \mathrm{gr}$ \\
\hline /1-NB & 4.0 & $5.035 \mathrm{~mm}$ \\
\hline (N-Pog).(Po-Orb) & $88.0 \pm 1.0$ & $90.361 \mathrm{gr}$ \\
\hline H-Nariz & $10.0 \pm 1.0$ & $5.342 \mathrm{~mm}$ \\
\hline
\end{tabular}

Fonte: CIMO - Clínica de Imagem (2021).

Após analisar os dados clínicos e cefalométricos, o plano de tratamento foi dividido em duas fases: fase 1 e fase 2 , como forma de priorizar a correção da mordida cruzada anterior e reduzir as discrepâncias esqueléticas observadas.

Para o início da fase 1 , foi realizada a instalação das bandas ortodônticas nos elementos 17 e 26 , foi realizada a moldagem com alginato (Ezact) para confecção dos modelos superior e inferior. Após uma semana foi instalado o expansor Haas com a finalidade de realizar a expansão transversal da maxila (figura 5) já sendo ativado 1 volta inteira após a instalação. Foi prescrita a ativação: 2/4 de volta pela manhã e 2/4 de volta pela noite, totalizando 1 volta inteira por dia durante 7 dias.

Durante a fase 2 será realizado o tratamento com aparelho 4x2 para o devido alinhamento e nivelamento da dentição permanente, assim que irrompidos.

Figura 5 - (A e B) Expansor Haas.

(A e B) Fotografias Extra bucais com máscara facial: A) Frontal; B) Lateral.
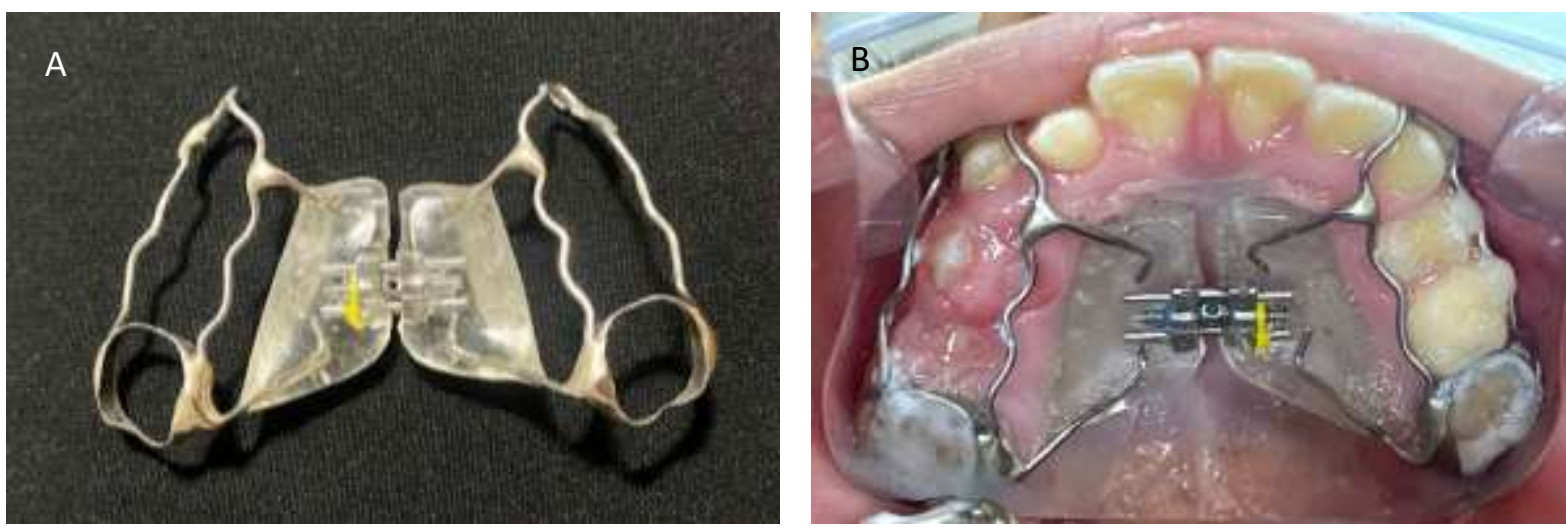

Fonte: Autores.

A expansão transversal foi satisfatória, da qual o alargamento da sutura palatina mediana, foi comprovado clinicamente com o aparecimento do diastema entre os elementos 11 e 21 e radiograficamente pela radiografia oclusal. A próxima etapa do tratamento, após a ERM, foi a tração reversa maxilar por meio da máscara facial de Petit, uma vez que a desarticulação das suturas circum-maxilares proporcionadas pelo procedimento prévio, potencializam o efeito de avanço maxilar e, consequentemente, melhoram a relação esquelética. 
Após a Expansão Rápida da Maxila (ERM), foi adaptada para a paciente, a máscara facial (figura 6). A instalação foi realizada com um elástico extra-oral $1 / 2$ pesado para ambos os lados no anel do meio da máscara, realizando a tração ortopédica da maxila. A paciente foi orientada a fazer o uso da máscara por no mínimo $16 \mathrm{~h}$ por dia.

Figura 6 - (A e B). Fotografias Iniciais Extra bucais com máscara facial: A) Frontal; B) Lateral.
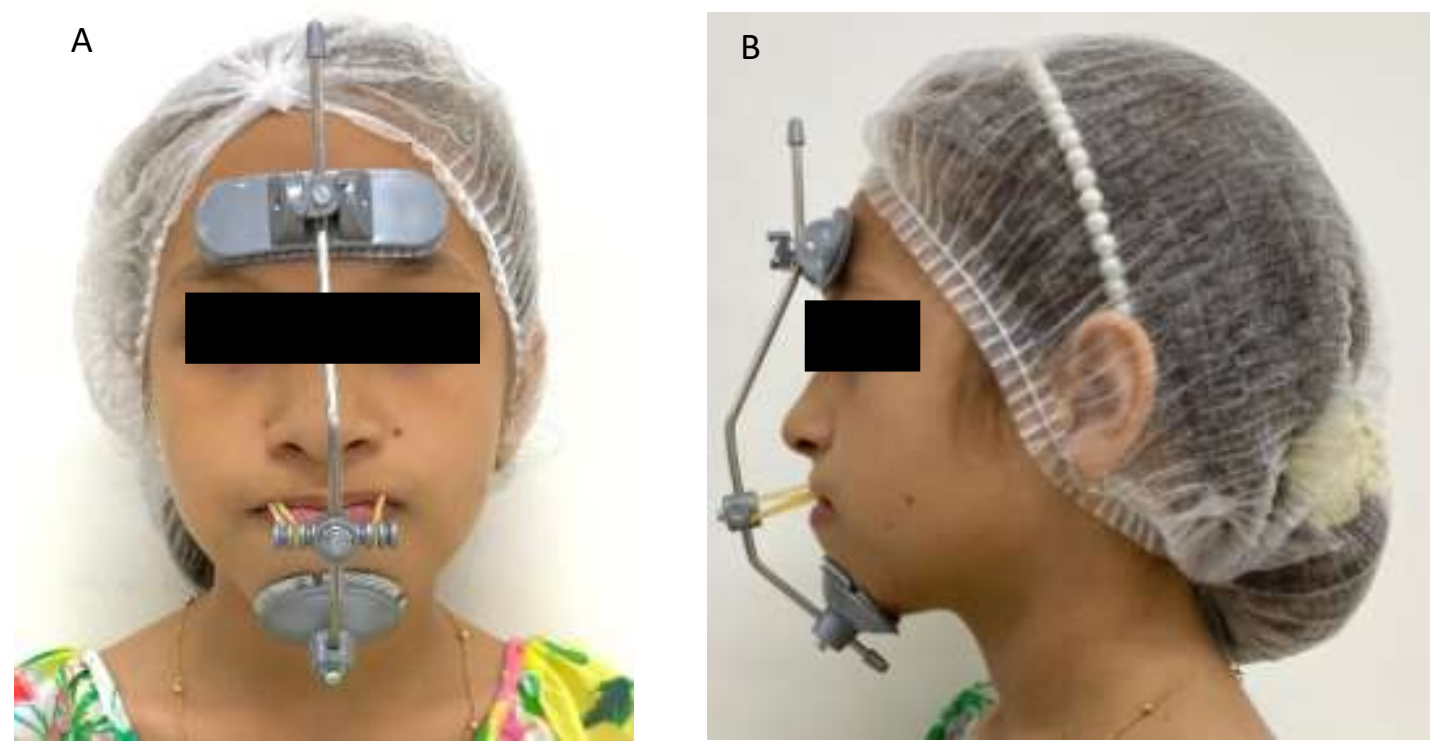

Fonte: Autores.

Após 2 meses, houve uma melhora significante na oclusão da paciente, observou-se o descruzamento da mordida (Figura 7) e foi realizada a manutenção da máscara, assim evoluindo o tratamento. 
Research, Society and Development, v. 11, n. 1, e29511124698, 2022

(CC BY 4.0) | ISSN 2525-3409 | DOI: http://dx.doi.org/10.33448/rsd-v11i1.24698

Figuras 7 - (A - D) Fotos intra orais com o disjuntor Haas e ganchos nos caninos para máscara facial de Petit, após dois meses de uso.
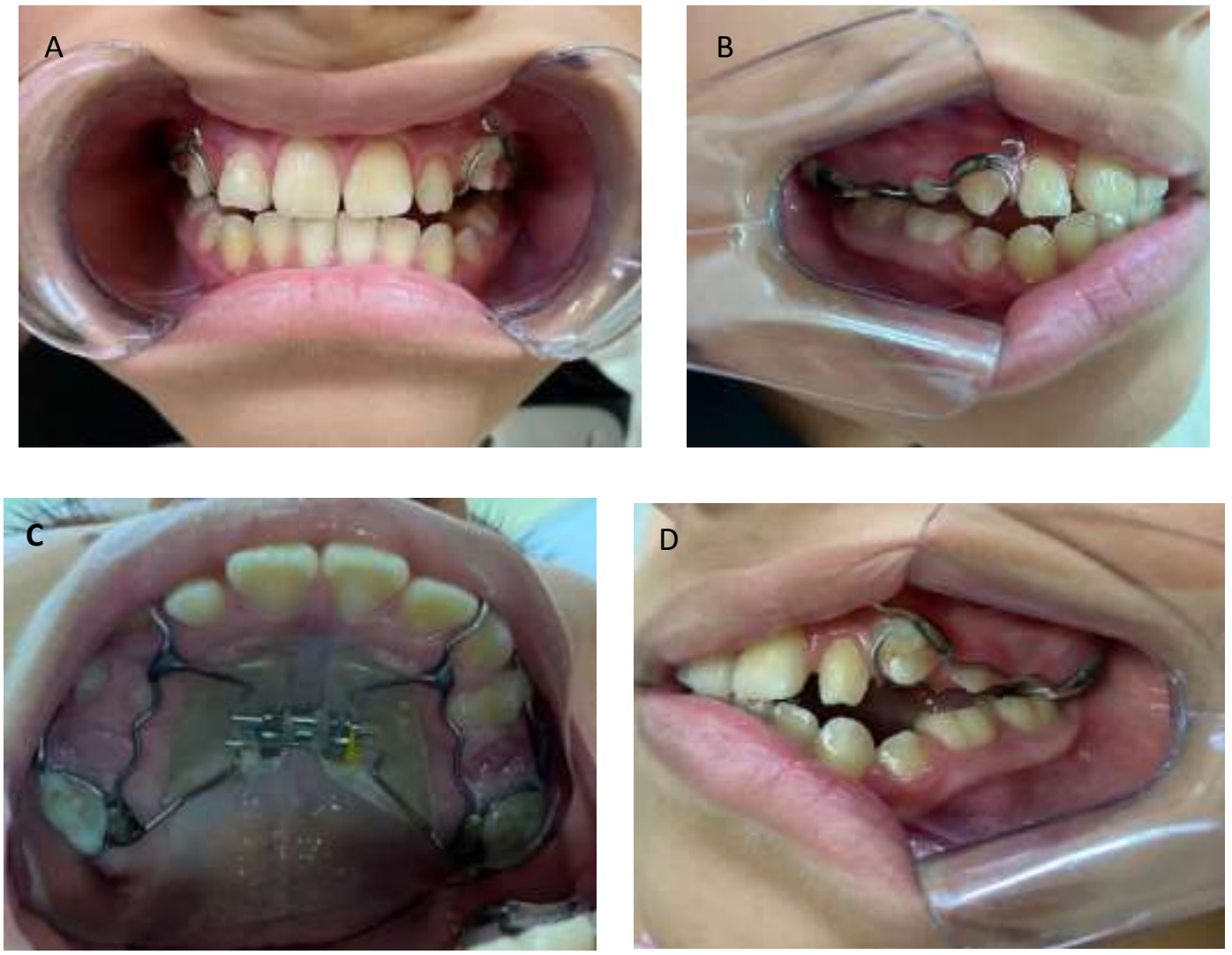

Fonte: Autores.

Nesta evolução foram usados 2 elásticos extraorais $1 / 2$ pesado para ambos os lados no anel do meio da máscara, com o auxílio do tênsiometro, foi possível identificar que estávamos com $500 \mathrm{~g}$ de força do lado direito e $520 \mathrm{~g}$ de força no lado esquerdo (Figura 8).

Figura 8 - (A e B) Fotografias Extra bucais com máscara facial: A) Frontal; B) Lateral.
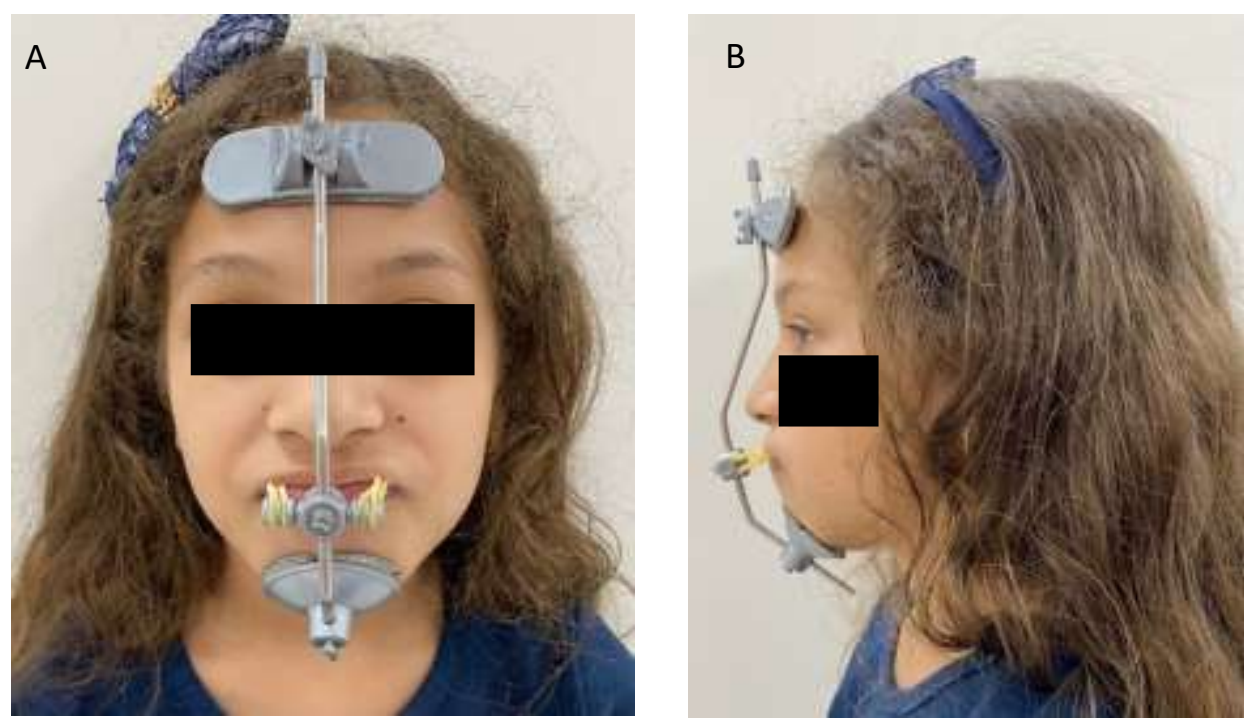

Fonte: Autores. 
Em decorrência do pouco tempo em tratamento da paciente, não foi possível relatar a finalização da intervenção, porém, a paciente ainda irá dar continuidade a terapia interceptativa até que todos objetivos traçados no plano de tratamento sejam concluídos.

\section{Discussão}

É comum as más oclusões de Classe III esqueléticas agravarem com os anos, por essa razão, o tratamento interceptativo é essencial em pacientes no período da dentição decídua ou mista, é importante um tratamento prévio. Esse tipo de tratamento, é um grande desafio para os especialistas por conta de sua complexidade (Melo et al., 2020).

É de suma importância o tratamento ortopédico de má oclusão, bem como as características físicas da oclusão é difícil padronizar julgamentos e comparações, além de proporcionar ao paciente a possibilidade de não se fazer necessário o tratamento cirúrgico na fase adulta, apesar que esta afirmação só poderá ser constatada ao fim do crescimento do paciente (Kaygisiz et al., 2016).

Segundo Ricardo Damo Meneguzzi (2019), a má oclusão de classe III de Angle pode ser definida como uma desarmonia sagital da face, caracterizada por uma protrusão mandibular, retrusão maxilar ou a combinação de ambas, frequentemente associada a mordida cruzada decorrente de uma atresia transversal da maxila (Meneguzzi, 2019). No presente caso, a paciente apresenta retrusão maxilar.

Os expansores Haas, Hyrax e o Mcnamara promovem a disjunção do palato. Somente no expansor tipo Haas há a presença de um componente de acrílico que provocam a inclinação em direção ás laterais do palato (Flores et al., 2021). O Haas e o Hyrax, são disjuntores palatinos que necessitam de bandagem ortodôntica e promovem a abertura da sutura palatina mediana, são escolhas efetivas para a terapia da assimetria transversal, e podem estar associados ganchos para a tração reversa (Meneguzzi, 2019).

Segundo estudos sobre os disjuntores tipo Hyrax e Haas, foi descrito que a maior diferença entre eles é a presença da estrutura em acrílico no disjuntor Haas (mucosuportado), tendo em vista que a ausência dessa estrutura no disjuntor Hyrax causa a vestibularização dos dentes posteriores durante a ativação (Izquierdo et al., 2020). O disjuntor palatino de escolha foi o Haas pois, não seria favorável para o resultado, a inclinação vestibular de dentes posteriores e por suprir as necessidades do tratamento.

O tratamento realizado precocemente da Classe III, permite melhoras no desenvolvimento da face, evitando complicações na articulação temporomandibular (ATM). A terapia necessita de ERM com o auxílio da máscara facial, a máscara facial promove a tração anterior da maxila, derivando em modificações esqueléticas e dentárias que resultam na melhora considerável do perfil facial. A realização desse tratamento tem indicação para pacientes a partir de 5 anos de idade, no período de crescimento, durante a fase da dentição decídua (Fernandes et al., 2019). A paciente iniciou o tratamento com nove anos de idade e apresentou um resultado vantajoso quanto a ERM associada à máscara facial.

De acordo com a literatura pesquisada, os especialistas em ortodontia afirmam que há um número considerável de crianças com má oclusão, ângulo de mandíbula anormal, mordida cruzada posterior e anterior, os tratamentos geralmente começam por volta dos sete anos de idade, porém, é notável a dismorfologia facial antes. Habitualmente as más oclusões esqueléticas de Classe III não são tratadas unicamente por técnicas intrabucais devido à dificuldade. Em virtude disso, os métodos de tratamento extrabucais tal como, a tração reversa da maxila é adequada para procedimentos em conjunto com técnicas intrabucais em ambos os períodos da dentição com o objetivo de eliminar a opção de correção cirúrgica futuramente. Para esse tipo de tratamento, a melhor escolha é a máscara facial, possui várias vantagens em especial na aparência física e harmonia (Suassuna et al., 2019). Este protocolo mostrou-se efetivo à paciente, sendo possível observar uma significante melhora na harmonia do sorriso após o início do tratamento com aparelho intrabucal sendo realizada a disjunção palatina associada à máscara facial. 
No presente caso, a paciente apresentou melhora significante na harmonia do sorriso após sete meses de tratamento. A paciente irá realizar a fase 2 do tratamento ortodôntico $4 \times 2$ como continuação da terapia ortodôntica. Conforme o presente relato, a paciente ainda está em acompanhamento.

\section{Considerações Finais}

A má oclusão de Classe III é um grande desafio para os ortodontistas, é fundamental uma atenção especial durante o diagnóstico e terapia proposta. Os estudos comprovam que há mais chances deste tipo de tratamento ser eficaz se for iniciado durante a dentição decídua ou mista, visto que o paciente ainda está em período de crescimento esquelético. O conjunto da expansão rápida da maxila associado à máscara facial de Petit é o tratamento de escolha para os autores, tendo em vista que os resultados são mais favoráveis na fase da dentição decídua ou mista, fatores genéticos e a colaboração da paciente e responsáveis também é essencial.

No caso em questão, a paciente continua em tratamento, tendo obtido o cumprimento da abertura da sutura palatina e a instalação da máscara facial. Após a finalização da fase 1, será necessário o tratamento ortodôntico com aparelho 4x2.

\section{Referências}

Alhammadi, M. S. et al. (2018). Global distribution of malocclusion traits: A systematic review. Dental Press Journal of Orthodontics. 23(6):40. e1-10.

Andrade, G. S., \& Bittencourt, M. A. V. (2021). Tratamento da mordida cruzada anterior esquelética: acompanhamento após quatro anos. Rev Fac Odontol Univ Fed Bahia. 51(2): 92-106.

Bedolla, H. et al. (2018) Quick correction of a skeletal class III maloclussion in primary dentition with face mask plus rapid maxillary expansion therapy. ODOVTOS-International Journal of Dental Sciences. 20(2):31-37.

Brunetto, D. P. et al. (2017). Non-surgical treatment of transverse deficiency in adults using Microimplant-assisted Rapid Palatal Expansion (MARPE). Dental Press Journal of Orthodontics. 22(1):110-125.

Cruz, J. H. A. et al. (2019). Mordida cruzada posterior: um enfoque à epidemiologia, etiologia, diagnóstico e tratamento. Archives of health investigation. $8(3): 157-163$.

Fernandes, N. L. F. et al. (2019). Mordida cruzada anterior: possibilidades de tratamento na dentição decídua e mista. Revista Naval de Odontologia. 46(1):5968 .

Freitas, B. G. et al. (2019). As vantagens do tratamento precoce da Classe III. Revista Faipe. 9(2):24-28.

Flores, R. P. et al. (2021). Expansão rápida da maxila. Revista Faipe. 11(1):25-40.

Imbaud, T. C. S. et al. (2016). Frequência de rinite e alterações orofaciais em pacientes com má oclusão dentária. Revista Paulista de Pediatria. 34(2):184-188. Izquierdo, L. A. V. et al. (2020). Eficácia dos aparelhos de expansão rápida da maxila Hyrax e Haas. J Multidiscipl Dent. 10(2):112-119.

Kaygisiz, E., Uzuner, F., \& Taner, L. (2016). A comparison of three orthodontic treatment indices with regard to angle classification. The Journal of Clinical Pediatric Dentistry. 40(2)169-174.

Lopes, C. L., Costa, J. V., \& Oliveira, R. C. G. (2015). Tratamento precoce da classe III com expansor de Haas associado à máscara facial de Petit - relato de caso clínico. Revista UNINGÁ Review. 24(2):26-33.

Machado, V. et al. (2019). A systematic review and meta-analysis on Bolton's ratios: Normal occlusion and malocclusion. Journal of Orthodontics. 47(1):7-29.

Melo T. R. N. B., Oliveira, L. A. D., \&, Diniz, M. A. G. (2020). Tração reversa da maxila: relato de caso clínico. Revista Eletrônica Acervo Odontológico. $1(3252) ; 1-7$.

Melo T. R. N. B., \& Souza, G. R. (2020). Avaliação da distância intercaninos e sua correlação com as más oclusões. Revista Eletrônica Acervo Odontológico. 1(3997):1-8.

Meneguzzi, R. D. (2019). Emprego de disjuntor palatino encapsulado na expansão rápida maxilar previamente à terapia de tração reversa da maxila: Relato de caso. Revista Odontológica do Hospital de Aeronáutica de Canoas. 1(1):33-38.

Morais, C. H. et al. (2016). Malocclusion in schoolhildren aged 7-12 years old in Minas Gerais, Brazil. RGO - Rev Gaúch Odontol. 64(2):164-170.

Oppitz, L. R. et al. (2021). Aesthetic and functional advantages of early orthodontic treatment in a child with Asperger's Syndrome: case report. Research, Society and Development, 10(15), e187101522722. https://doi.org/10.33448/rsd-v10i15.22722 
Research, Society and Development, v. 11, n. 1, e29511124698, 2022

(CC BY 4.0) | ISSN 2525-3409 | DOI: http://dx.doi.org/10.33448/rsd-v11i1.24698

Peclat, M. M. S., Silva, V. T., \& Oliveira, T. C. P. (2017). A utilização da tração reversa no tratamento da maloclusão de Classe III de Angle: uma revisão de literatura. Rev Nav Odontol. 44(1):5-9.

Suassuna, K. M. L. et al. (2019). Expansão e disjunção palatina em pacientes classe III com uso de máscara facial. Revista de Odontologia da Universidade Cidade de São Paulo. 30(3):290-303.

Zou, J., Meng, M., Law, C. S., Rao, Y., \& Zhou, X. (2018). Common dental diseases in children and malocclusion. International Journal of Oral Science. 10(1), 7. https://doi.org/10.1038/s41368-018-0012-3. 